\title{
THEMATIC
} PAPER

\section{The Telepsychiatry Operational Guidelines 2020 in India: a welcome step}

\author{
Kumari Rina, ${ }^{1}$ @ Susanta Kumar Padhy ${ }^{2} \odot$ and Rakesh Kumar Chadda ${ }^{3} \odot$
}

MD, Assistant Professor Department of Psychiatry, Al Sciences, Bhubaneswar, India ${ }^{2} \mathrm{MD}$, Additional Professor and Head, Department of Psychiatry, All India Institute of Medical Sciences, Bhubaneswar, India. Email psych_susanta@aiimsbhubaneswar.edu.in

${ }^{3}$ MD Psychiatry, FRCPsych, Professor and Head, Department of Psychiatry, All India Institute of Medical Sciences, New Delhi, India

Keywords. Telemedicine guidelines 2020; telepsychiatry guidelines 2020; telepsychiatry;

First received 7 Oct 2020 Final revision 14 Jan 2021

doi:10.1192/bji.2021.20

The Author(s), 2021. Published by Cambridge University Press on behalf of the Royal College of Psychiatrists. This is an Open Access article, distributed under the terms of the Creative Comms NonCommercial-NoDerivatives licence (http://creativecommons. org/licenses/by-nc-nd/4.0/), which permits non-commercial re-use, distribution, and reproduction in any medium, provided the origin ginal work is unaltered properly cited. The written permission of Cambridge University Press must be obtained for commercial re-use or in order to create a derivative work.
The release of the Telepsychiatry Operational Guidelines 2020 in India, during the COVID-19 pandemic, is a relief for both clinicians and patients. These guidelines embark on initiating and executing naive and recently started telepsychiatry services in India. The document is aligned with other ethical regulations, policies, laws and the $\mathbf{2 0 2 0}$ Telemedicine Practice Guidelines in India. This paper discusses a few points about the broader applicability of the guideline for the benefit of humankind in the prevailing healthcare crisis. The guidelines may be extrapolated in policy-making for telepsychiatry services in other low- and middle-income countries sharing a similar socioeconomic, cultural and political milieu.

The COVID-19 pandemic has played havoc with the delivery of healthcare services, with diversion of services from general medical care to COVID-specific care. Persons with mental illness are vulnerable owing to the typically chronic nature of illness and inability for self-care. Telemedicine guidelines by the Board of Governors of the Medical Council of India and introduction of the Telepsychiatry Operational Guidelines 2020 by the Indian Psychiatric Society, the Telemedicine Society of India and the National Institute of Mental Health and Neuro Sciences (NIMHANS) have been a welcome and proactive development. It is worthwhile mentioning that these guidelines have improved the horizons for mental healthcare in India. ${ }^{1}$ The telepsychiatry guidelines inform psychiatrists on starting, establishing, executing and maintaining the provision of telepsychiatry services in any organisation, institute, clinic or hospital. The document takes into account and matches with other ethical regulations in India, such as the Information Technology Act 2000, the Drugs and Cosmetics Act 1940, the Rights of Persons with Disability Act 2016, the Mental Healthcare Act 2017 and the Narcotic Drugs and Psychotropic Substances Act 1985. It is also aligned with various other policies, laws and the 2020 Telemedicine Practice Guidelines.

The guidelines divide medicines that can be prescribed via tele-consultation into four categories (lists $\mathrm{O}, \mathrm{A}, \mathrm{B}$ and $\mathrm{C}$ ). List $\mathrm{O}$ drugs are over-the-counter medications and List $\mathrm{C}$ are prohibited for online prescribing. List A medicines are relatively safe and have low potential for misuse. For prescribing medications under list $\mathrm{A}$, a live and simultaneous video consultation is mandatory for the first/new consultation. For tele-follow-up consultation, any mode (audio/ text/video) is permitted for medicine refill. List B medicines are described as 'add-on' medicines employed to optimise a psychiatric condition. List B medicines can be prescribed in telefollow-up consultation only and any mode (audio/video/text) can be used for the consultation. A tele-follow-up consultation means that the patient is consulting with the same clinician, for the same health condition within 6 months of a previous in-person consultation. ${ }^{1}$ Thus, for prescribing list $\mathrm{B}$ medicines, there has to be at least one face-to-face consultation. Of course, it is good that the patient has to visit only once and subsequent visit frequency decreases significantly (at least in some cases). The benefits of prescribing list-B medicines after one face-to-face consultation, vis-a-vis visiting a hospital and not complying to lockdown measures, might appear unreasonable. To the best of our knowledge, there is no comparative study to answer the query. Let us try to analyse it with the available evidence.

\section{Curtailing clinicians' prescribing choices}

Among the antidepressants, list A includes only escitalopram and fluoxetine; the others are on list B. However, the evidence says that, among 21 antidepressants for the acute treatment of adults with major depressive disorder, escitalopram, paroxetine, amitriptyline, mirtazapine, venlafaxine, vortioxetine and agomelatine are more effective than other antidepressants, whereas fluvoxamine, trazodone, fluoxetine and reboxetine are the least efficacious. Tolerability and acceptability are higher for escitalopram, sertraline, citalopram, fluoxetine, agomelatine and vortioxetine than for the other antidepressants. Drop-out rates are higher for fluvoxamine, amitriptyline, clomipramine, venlafaxine, duloxetine, trazodone and reboxetine than for other antidepressants. ${ }^{2}$

The parallel is the case with antipsychotics. List A antipsychotics are risperidone, olanzapine and haloperidol only, while rest are under list B. ${ }^{1}$ Although in a given patient there may be some clinically meaningful differences in response due to individual differences in biological constitution and behaviour, to date there are no conclusive data that one second-generation antipsychotic will have superior efficacy over another (with clozapine in treatment-resistant schizophrenia as an exception). ${ }^{3} \quad$ A recent network meta-analysis of 
head-to-head randomised placebo-controlled trials comparing 32 antipsychotics found only minor efficacy differences between antipsychotics. There are marked differences among antipsychotics in terms of side-effects. ${ }^{4}$ The right choice of an antipsychotic medication and the route of administration should be based on weighing the patient's previous experience with antipsychotics, the side-effect profile, response to symptoms and the patient's preference for a particular medication. ${ }^{3,4}$

Let us ponder a possible clinical scenario where the patient has a history (more than 6 months) of good response to antidepressants other than escitalopram or fluoxetine or to antipsychotics other than risperidone, olanzapine and haloperidol; or a history of failed trials of escitalopram and fluoxetine for depressive disorders or list A antipsychotics for psychotic disorders/ schizophrenia. The guidelines' categorisation of psychotropics curtails the clinicians' freedom to select the best medication for that patient.

Among anti-epileptic drugs, list A contains only phenobarbitone, diphenylhydantoin and sodium valproate, and there is only divalproex sodium on list B. A point to be noted here is that the list A medications are readily available, and hence keeping them on list A makes sense. On the other hand, data reflect that for treating generalised tonic-clonic, tonic and clonic seizures, levetiracetam, lamotrigine and topiramate are as effective as valproate. For absence seizures, valproate and ethosuximide are the best available treatment options. ${ }^{5}$ So, allowing only four antiepileptics restricts the clinical choice of clinicians. Sodium valproate is on list $\mathrm{A}$, whereas divalproex sodium is on list B. Each formulation of valproate is therapeutically equivalent because, at physiological $\mathrm{pH}$, valproic acid dissociates into valproate ions. ${ }^{6}$ Placement of the two preparations on different lists may not be warranted.

\section{Is 'add-on' a misnomer?}

As mentioned above, the medications on list B are described as 'add-on' medications, prescribed to optimise a particular psychiatric illness. List B antipsychotics such as aripiprazole, quetiapine, amisulpride and trifluoperazine have similar efficacy to olanzapine, risperidone and haloperidol, which are on list A. List B antidepressants and antipsychotics are similar to those on list A in terms of efficacy but differ in their tolerability. ${ }^{2,4}$ Thus, calling list B 'add-on' medications appears a misonmer.

If a psychiatrist is generating a prescription for injectable haloperidol, fluphenazine or promethazine in an online consultation with a registered medical practitioner, then these injectables are considered to be under list A, whereas in the same situation zuclopenthixol, flupentixol, paliperidone and aripiprazole are under list B. First-generation depot antipsychotics are cost-effective but secondgeneration depots are better tolerated, ${ }^{7}$ but this is not reflected in their listing under this system. Moreover, depot olanzapine, considering its costbenefit analysis, could be a list A medication.

\section{Other drugs}

It is not clear whether methylphenidate, a firstline agent in the management of attention-deficit hyperactivity disorder (ADHD), can be teleprescribed. It might be assumed that it is falls under 'other drugs' in list B (under anti-ADHD drugs). However, methylphenidate is listed in the Narcotic Drugs and Psychotropic Substances Act 1985 (NDPS). If we consider that it is approved under list $\mathrm{B}$, an argument may arise that one drug under the NDPS has been approved whereas others (list C) are not. List C (teleprescription prohibited) contains drugs under schedule $\mathrm{X}$ of the Drugs and Cosmetics Act 1940 and Drugs and Cosmetics Rules 1945 and substances listed in the NDPS - morphine, buprenorphine, methadone, benzodiazepines (except clobazam and clonazepam!), Z-drugs such as zolpidem, and so on. Many high-income countries, the USA for example, have relaxed the rules to allow psychiatrists to evaluate patients via telephone or video calls before prescribing these medications. ${ }^{8}$ India has twice the global average prevalence of illicit opioid consumption, with 23 million users. ${ }^{9}$ A handful of them seek medical help. With such an inclusion, the barrier to help-seeking by people with substance use disorder may be magnified.

\section{Psychotherapy}

For psychotherapy, the ethics, legal provisions, accountability and responsibility requirements for teletherapy are similar to those for face-to-face therapy. Since the arrival of the pandemic on the Indian subcontinent, medical professionals have presented numerous virtual sessions for promotion of positive mental health, stress management, coping with the pandemic and similar issues. Nonetheless, as teletherapy carries certain limitations and therefore the guidelines advise a detailed initial face-to-face assessment. Even in detailed face-to-face assessment, a mask on the face of both the patient and the doctor impedes rapport, the transmission of empathy, the therapeutic alliance and outcome. ${ }^{10}$ Such circumstances may be a barrier for some people who would like to choose psychotherapy as a modality of treatment. Of course, there are exceptions behavioural therapies, for example, might be less affected by mask-wearing and tele-delivery.

\section{Suicide and self-harm}

Recent literature suggests that the number of people contemplating suicide and self-harm in India is rising. ${ }^{11}$ Of course, modified electroconvulsive therapy (ECT) is the treatment of choice where suicidal intent is high. ${ }^{12}$ But the silence of these guidelines on telepsychiatry for people who present with suicide and self-harm should not create uncertainty among clinicians if the clinical situation calls for its use. 


\section{Conclusions}

Owing to the suddenness of lockdown, many patients were underprepared for the management of their illness and stock of medications. There are reports of poor compliance owing to availability problems. We therefore suggest that the choice of psychotropic in telepsychiatry consultations should be left to the treating psychiatrist based on clinical judgement and drug regulations. Similarly, some psychotherapeutic interventions should be permitted in telepsychiatry. Of course, these guidelines are intended to guide clinicians in patient care, not to dictate what must be done. They undoubtedly offer a buffer for clinicians during this period of healthcare crisis. Moreover, they may help other low- and middle-income countries of similar socioeconomic, cultural and political background with limited psychiatrists and other resources in policy reform for telepsychiatry services, drug regulation and efficient delivery of mental healthcare.

\section{Data availability}

Data availability is not applicable to this article as no new data were created or analysed in this study.

\section{Author contributions}

K.R. planned and drafted the paper. S.K.P. conceived the idea, provided critical revision of the article for intellectual content and final approval of the version to publish. R.K.C provided critical revision of the article for intellectual content and final approval of the version to publish.

\section{Funding}

This research received no specific grant from any funding agency, commercial or not-for-profit sectors.

\section{Declaration of interest}

\section{None.}

\section{References}

1 Math SB, Manjunatha N, Kumar CN, Basavarajappa C, Gangadhar BN. Telepsychiatry Operational Guidelines - 2020 National Institute of Mental Health and Neuro Sciences, 2020.
2 Cipriani A, Furukawa TA, Salanti G, Chaimani A, Atkinson LZ, Ogawa Y, et al Comparative efficacy and acceptability of 21 antidepressant drugs for the acute treatment of adults with major depressive disorder: a systematic review and network meta-analysis. Lancet 2018; 391: 1357-66.

3 Lehman AF, Lieberman JA, Dixon LB, McGlashan TH, Miller AL, Perkins DO, et al Practice guideline for the treatment of patients with schizophrenia, second edition. Am J Psychiatry 2004; 161 (suppl 2): 1-56.

4 Huhn M, Nikolakopoulou A, Schneider-Thoma J, Krause M, Samara M, Peter N, et al Comparative efficacy and tolerability of 32 oral antipsychotics for the acute treatment of adults with multi-episode schizophrenia: a systematic review and network meta-analysis. Lancet 2019; 394: 939-51.

5 Sadock B], Sadock VA. Kaplan and Sadock's Synopsis of Psychiatry: Behavioral Sciences/Clinical Psychiatry 10th edn. Lippincott Williams and Wilkins, 2007.

6 de Almeida Campos MS, Ayres LR, Morelo MR, Carizio FA Pereira LR. Comparative efficacy of antiepileptic drugs for patients with generalized epileptic seizures: systematic review and network meta-analyses. Int J Clin Pharm 2018; 40: 589-98.

7 Whyte A, Parker C. A review of the efficacy and tolerability of antipsychotic long-acting injections. Prog Neurol Psychiatry 2016; 20: 22-8.

8 Conolly B. Federal government eases access to opioid use disorder treatment during pandemic. Pew Charitable Trusts, 2020 (https://www.pewtrusts.org/en/research-and-analysis/ articles/2020/04/01/federal-government-eases-access-to-opioiduse-disorder-treatment-during-pandemic [cited 24 Jul 2020]).

9 Ambekar A, Agrawal A, Rao R, Mishra AK, Khandelwal SK, Chadda RK, et al Magnitude of Substance Use in India. Ministry of Social Justice and Empowerment, Government of India, 2019.

10 Wong CK, Yip BH, Mercer S, Griffiths S, Kung K, Wong MC, et al Effect of facemasks on empathy and relational continuity: a randomised controlled trial in primary care. BMC Fam Pract 2013; 14: 200.

11 Joseph JP. COVID-19 and mental health: suicidal tendencies and self-harm on the rise. The Wire, 2020 (https://thewire.in/health/ covid-19-mental-health-suicidal-tendencies-self-harm-rise [cited 27 Jul 2020]).

12 Fink M, Kellner $\mathrm{CH}$, McCall WV. The role of ECT in suicide prevention. J ECT 2014; 30: 5-9. 